\title{
BRECHAS REGIONALES DE LA MORTALIDAD INFANTIL EN COLOMBIA
}

\author{
Marta C. Jaramillo-Mejía1,a, Dov Chernichovsky²,b, José J. Jiménez-Moleón ${ }^{3,4, c}$
}

RESUMEN

Objetivos. Estudiar las variaciones de la tasa de mortalidad infantil (TMI) en los departamentos de Colombia durante el período 2003-2009, examinar la persistencia de las variaciones entre los departamentos sobre el tiempo y relacionarlas con el impacto de las condiciones socioeconómicas y la disponibilidad de servicios de salud, sobre la mortalidad infantil. Materiales y métodos. Utilizando estadísticas vitales y relacionando datos socioeconómicos y de servicios de salud, se analizaron tres aspectos: la variación de la TMI departamental (2003-2009), la relación entre la TMI departamental y determinantes claves en el tiempo, y las líneas de causalidad e impacto relativo de los diferentes factores. Se emplearan ecuaciones estructurales. Resultados. Se encontró una razón de 4,7 entre la mayor y menor TMI departamental (2009), esta podría estar subestimada principalmente por el subregistros en departamentos de bajos ingresos. Hay una relación negativa entre la TMI departamental con el tiempo y variables altamente correlacionadas, como educación de la madre, ingreso per cápita, cobertura de aseguramiento y acceso a servicios. Conclusiones. El efecto del aseguramiento, disponibilidad de camas privadas y atención médica, es superior al impacto de mejores condiciones socioeconómicas sobre la TMI. La oferta de servicios no parece estar influenciada por una política racional, los recursos no se asignan de acuerdo con las necesidades, sino con el desarrollo general. Las camas privadas se hacen disponibles donde hay mejor aseguramiento en salud y menor TMI.

Palabras clave: Tasa de mortalidad infantil; Desigualdades en la salud; Factores socioeconómicos; Cobertura del seguro; Colombia (fuente: DeCS BIREME).

\section{REGIONAL DISPARITIES IN INFANT MORTALITY IN COLOMBIA}

\section{ABSTRACT}

Objectives. To study the variations in infant mortality rate (IMR) across Colombia's 33 administrative departments over the period 2003-2009, examine persistency of variations across departments over time, and relate those variations to the impact of socio-economic conditions and availability of care on IMR. Materials and methods. Using vital statistics and related socio-economic data we establish three types of analysis according to: (a) the variation of the departmental IMR (20032009), (b) the association between the departmental IMR and its key determinants over time, and (c) the lines of causality and relative impact of different factors, by using structural equations. Results. The 4.7 fold ratio between the highest and lowest departmental IMR (2009) may be underestimated considering underreporting, especially in low-income departments. There is a negative association between the departmental IMR with time and a set of highly correlated variables, such as the mother education, income per capita, health insurance level and access to services. Conclusions. The effect of better insurance, availability of private beds, and having doctors attending mothers, eclipse the impact of better socioeconomic conditions. The range of services does not appear to be influenced by a rational policy; resources are not allocated according to the need, but with the general development. Private beds are made available where there is better health insurance.

Key words: Infant mortality rate; Health inequalities; Socioeconomic factors; Insurance coverage; Colombia (source: MeSH NLM).

\section{INTRODUCCIÓN}

La tasa de mortalidad infantil (TMI) en Colombia, estimada para 2009, es de 17,2 muertes por mil nacidos vivos (NV) ${ }^{(1)}$, que resulta de conjugar varias fuentes, considerando que la tasa registrada es 13,7 y la tasa estimada por el Departamento Administrativo Nacional de Estadística (DANE) es de 20,1 por mil NV ${ }^{(2,3)}$. La TMI colombiana es mayor que la tasa media de 16,2 estimada para América Latina y el Caribe y del grupo de países

\footnotetext{
Departamento de Gestión Organizacional, Universidad Icesi. Cali, Colombia.

Department of Health Systems Administration. Ben Gurion University of the Negev. Beer-Sheva, Israel.

Departamento de Medicina Preventiva y Salud Pública, Facultad de Medicina, Universidad de Granada. Granada, España.

Consorcio de Investigación Biomédica de Epidemiología y Salud Pública (CIBERESP). Granada, España.

Médico, máster en Investigación y avances en Medicina Preventiva y Salud Pública; ${ }^{\mathrm{b}}$ economista, PhD en Economía; ${ }^{\mathrm{c}}$ médico, PhD en Medicina.

* Los resultados del presente estudio forman parte de la Tesis de Marta Cecilia Jaramillo-Mejía, para optar al grado de PhD en Medicina Clínica y Salud Pública de la Universidad de Granada, España; con la Dirección de los doctores Dov Chernichovsky y José Juan Jiménez-Moleón.

Recibido: 15-10-2013 Aprobado: 06-11-13
}

Citar como: Jaramillo-Mejía MC, Chernichovsky D, Jiménez-Moleón JJ. Brechas regionales de la mortalidad infantil en Colombia. Rev Peru Med Exp Salud Publica. 2013;30(4):551-9. 
con niveles de ingreso similar a Colombia. Además, es considerablemente más alta que la tasa de Chile (7,9 en 2009), a pesar de que ambos países compartieron una TMI similar en $1950^{(4,5)}$. Sin embargo, el promedio de la tasa colombiana es relativamente bajo y no refleja la alta disparidad regional en las tasas a través de los 1141 748 kilómetros cuadrados que albergan alrededor de 46 millones de personas (2012) ${ }^{(6)}$.

El sistema de salud colombiano, establecido por la Ley 100 en $1993^{(7)}$, está conformado por dos regímenes: el régimen contributivo $(\mathrm{RC})$ con una cobertura cercana al $40 \%$, para la población en la economía formal y el régimen subsidiado (RS), que cubre el $53 \%$ de la población más pobre (2010-2011). Los miembros del $\mathrm{RC}$ reciben un paquete de beneficios estipulado por el Plan Obligatorio de Salud (POS). Los afiliados al régimen subsidiado han recibido, por años, un paquete con menos beneficios, que solo se igualó a partir de $2013^{(8,9)}$

Es necesario, entonces, generar información con suficiente sustento científico que ayude a la formulación de políticas públicas en Colombia, a fin de reducir la TMI del país, mediante el estudio de las correlaciones entre las tasas de los 33 departamentos (división administrativa territorial -provincias-). El desafío de los estudios analíticos, de la misma naturaleza que el presente, es descomponer estadísticamente el impacto de la prestación de los servicios de salud sobre la TMI; desde las condiciones socioeconómicas, altamente correlacionadas con estos factores. Adicionalmente, es necesario estudiar los cambios según su importancia relativa en el tiempo. El reto es universal, pero es relativamente importante para países en transición como Colombia, donde las brechas interregionales en salud son amplias, y las características que definen el desarrollo están altamente correlacionadas con mediciones de la salud a través de las regiones, y tienden a permanecer en el tiempo. Es por ello que los objetivos del presente estudio son estudiar las variaciones de la TMI en los 33 departamentos administrativos de Colombia durante el periodo 2003-2009, examinar la persistencia de esas variaciones entre departamentos en el tiempo y relacionarlas con el impacto de las condiciones socioeconómicas y la disponibilidad de servicios de salud sobre la mortalidad infantil.

\section{MATERIALES Y MÉTODOS}

Se realizó un estudio ecológico. La base de datos empleada para el análisis proviene de tres fuentes combinadas. La primera contiene las estadísticas vitales (que comprenden los registros individuales de nacidos vivos y las muertes de niños menores de un año de edad), agrupadas a nivel departamental, procedentes del DANE e incluye datos sobre condiciones demográficas y socioeconómicas de la madre y el niño, en particular el nivel de educación y tipo de régimen de seguro médico, junto con variables asociadas al uso de intervenciones médicas y de servicios de salud. En la segunda se encuentran registrados los datos del ingreso anual per cápita de cada departamento, disponible en las cuentas nacionales y departamentales del DANE ${ }^{(10)}$. La tercera incluye las características de la infraestructura de los servicios de salud de cada departamento, el número de camas públicas y privadas materno-infantil (CMI) y de cuidados intensivos pediátricas y neonatales (CPN), provenientes del Registro Especial de Prestadores de Servicios de Salud (REPS) del Ministerio de Salud y Protección Social (MSPS), desde $2003^{(11) .}$

Con base en esta información, el análisis hace uso de las siguientes variables (cada una, excepto la última, medida para cada departamento y cada año): la tasa de mortalidad infantil $(M)$; la edad promedio de la madre $(A)$; el porcentaje de madres con educación secundaria o superior (E); el producto interno bruto (PIB) per cápita, en millones de pesos colombianos corrientes -en septiembre de 2013, un dólar americano correspondía a 1948 pesos colombianos- (Y); el porcentaje de madres en Régimen Contributivo $(I)$; el total de CMI por mil NV en el municipio de residencia de la madre $(B)$; el porcentaje de CMI privadas en el municipio de residencia $(P)$; el porcentaje de partos por cesárea $(C)$; el porcentaje de madres atendidas por médico $(D)$; y la tendencia temporal en años (en escala de -3 a 3 para el periodo 2003-2009) (T).

A partir de estas variables, los datos se someten a tres tipos de análisis que tienen como objetivo: describir la variación de la TMI departamental durante el período 2003-2009, destacando posibles sesgos en los datos; examinar la asociación entre la mortalidad infantil y las variables clave en todos los departamentos en el tiempo, para examinar la importancia relativa de sus variaciones; y determinar las posibles líneas de causalidad entre las variables, para estudiar aún más la importancia de los servicios médicos.

Para el primer análisis se empleó estadísticos descriptivos univariados en las tasas de descenso de la TMl departamental. Este método se basa en una relación de cambio exponencial, la cual suele describir la tendencia para variables demográficas. En este sentido, las tasas de descenso se estiman a partir de una media geométrica, de forma que la TMI para 2009 pueda expresarse en términos de la TMI para 2003 por una constante $\left(T M I_{2009}=T M I_{2003} \cdot g^{6}\right)$ Tal que $\mathrm{R}=100 \cdot(g-1)$ 
es la variación porcentual anual promedio. Esto permite estimar la tasa de descenso anual como una constante a lo largo del periodo de estudio.

Lo anterior es complementado por el segundo análisis que usa una regresión por mínimos cuadrados ordinarios (MCO) para la estimación de la función: $\ln \left(M_{i}\right)+\alpha+\beta \ln \left(X_{j}\right)+\varepsilon_{i}$ donde indistintamente, es una variable independiente seleccionada $-E$, I y $P$ - para el departamento en el momento y es un término aleatorio de error. Las variables seleccionadas tienen como objetivo establecer asociaciones entre la y, primero por separado: condiciones socioeconómicas, acceso potencial a los servicios superiores, y el uso real de atención de calidad. La doble especificación logarítmica sugiere una relación funcional no lineal del tipo $M=\alpha X^{\beta}$ donde $\alpha$ y $\beta$ son los parámetros a estimar. Entonces, $\beta$ es interpretada como la tasa de variación en la TMI a partir de un cambio porcentual en $X$. Las estimaciones se aplican para tres años por separado (2003, 2006 y 2009) para examinar efectos diferenciales de las variables en el tiempo.

El tercer análisis tiene por objeto establecer las líneas de causalidad, así como el impacto relativo de los diferentes factores, principalmente para ver si las condiciones socioeconómicas tienen un efecto directo sobre la TMI o a través del impacto de la disponibilidad de atención médica. A continuación se presenta el sistema de asociaciones considerado: (1) $\widehat{M}=\mathrm{m}(\widehat{C}, \widehat{D}, \mathrm{E})$; (2) $\widehat{C}=c($ $\widehat{B}, A, E, \widehat{P}) ;(3) \widehat{D}=d(\widehat{B}, \widehat{P}) ;(4) \hat{I}=i(Y, T) ;(5) \widehat{B}=b(\widehat{M}, \hat{I}, T)$; y (6) $\widehat{P}=b(\hat{I}, E)$ Las variables "resaltadas con gorro" son endógenas, en el sentido que son determinadas dentro del sistema a partir de unas variables exógenas. Las líneas de causalidad establecidas por las hipótesis plausibles son como siguen.

La mortalidad infantil (ecuación 1), se asume condicional a si los partos son asistidos por el médico (D); si se trata de un procedimiento por cesárea (C), lo cual representa la disponibilidad de atención de calidad y la situación socioeconómica de la madre, medida a través de la educación (E). En vista de la aparente falta de desnutrición materna en Colombia hoy en día, $E$ representa el acceso a mejores servicios, así como un mejor cumplimiento del cuidado prenatal de las madres relativamente más educadas y prósperas. Una cirugía cesárea (ecuación 2) se determina a partir de la edad de la madre $(A)$, indicando un riesgo que puede requerir del procedimiento; la disponibilidad total de CMI (B); el nivel de educación de la madre $(E)$, representando la demanda por el procedimiento y el porcentaje de CMI privadas $(P)$, sugiriendo que, cuando tales camas están disponibles, la demanda de cesáreas podría satisfacerse más fácilmente. Así mismo, la disponibilidad de médicos (ecuación 3), se considera que es influenciada por la infraestructura, medida en términos de disponibilidad de camas $(B)$, especialmente privadas $(P)$. La demanda por mejor aseguramiento (ecuación 4), se supone a su vez, que depende de la renta $(Y)$, así como del desarrollo general, aproximado por la tendencia temporal (T). La disponibilidad total de CMI se asume que es condicionada (ecuación 5) por una política racional, el suministro de camas es mayor en donde hay mayor necesidad o donde la mortalidad infantil (M) es relativamente alta. Además, es afectada por el acceso a un mejor aseguramiento (I), lo cual representa una generación de demanda por hospitalizaciones y por el desarrollo general y el crecimiento económico $(\mathrm{T})$. Por último, el porcentaje de CMI privadas (ecuación 6 ) se asume que es afectado por el acceso a mejor aseguramiento (I), a partir de la creación de la demanda de hospitalizaciones y por el nivel de educación de la madre $(E)$.

Para la estimación de los coeficientes de las ecuaciones estructurales utilizamos el método de mínimos cuadrados en tres etapas (MC3E) para todos los departamentos, en todos los años desde 2003 a $2009^{(10)}$. Este enfoque tiene la característica de datos de panel, mediante el cual se pueden identificar las variaciones entre departamentos con estimadores más precisos y eficientes. Este análisis estadístico asume independencia entre departamentos, algo razonable considerando que estos funcionan como entes administrativos independientes en Colombia. Este supuesto es respaldado por los datos en el primer y segundo análisis, donde se evidencian variaciones considerables en la TMI entre departamentos para cada año.

Por otra parte, dada la estructura de los datos, es posible encontrar correlación serial entre observaciones. Estos efectos son, sin embargo, capturados al incluir $T$ como variable independiente, por lo que no se espera que persista la posibilidad de un sesgo de este estilo. No obstante, al combinar datos a nivel espacial y temporal, se requiere asumir que los coeficientes de cada ecuación sean iguales entre departamentos. De lo contrario, se podría incurrir en un sesgo por heterogeneidad, en cuanto a condiciones socioeconómicas y de infraestructura en salud, entre departamentos.

\section{RESULTADOS}

El promedio de la TMI por departamento, registrada por las estadísticas vitales en Colombia, disminuyó de 17,0 \pm 4,0 (Coef. Variación: 0,2) por cada mil NV en 2003 a 13,6 \pm 3,9 (Coef. Variación: 0,3) en 2009, es decir, una tasa de reducción anual de 3,7 \pm 0,2 (Coef. Variación: 3,6) por ciento (Tabla 1); pero con rangos de variación desde una 
Tabla 1. Nivel y variación de la tasa de mortalidad infantil en Colombia, 2003 y 2009

\begin{tabular}{|c|c|c|c|}
\hline \multirow[t]{2}{*}{ Departamento } & \multicolumn{2}{|c|}{$\begin{array}{c}\text { Tasa de mortali- } \\
\text { dad infantil }\end{array}$} & \multirow{2}{*}{$\begin{array}{c}\text { Tasa de } \\
\text { variación } \\
\text { anual (\%) }\end{array}$} \\
\hline & 2003 & 2009 & \\
\hline Antioquia & 14,9 & 11,5 & $-4,2$ \\
\hline Atlántico & 20,8 & 14,6 & $-5,7$ \\
\hline Bogotá & 15,1 & 12,1 & $-3,6$ \\
\hline Bolívar & 22,0 & 13,7 & $-7,6$ \\
\hline Boyacá & 15,8 & 11,5 & $-5,2$ \\
\hline Caldas & 16,2 & 12,4 & $-4,4$ \\
\hline Caquetá & 29,3 & 19,5 & $-6,5$ \\
\hline Cauca & 20,1 & 18,8 & $-1,1$ \\
\hline Cesar & 19,6 & 16,7 & $-2,6$ \\
\hline Córdoba & 21,6 & 19,9 & $-1,4$ \\
\hline Cundinamarca & 17,5 & 11,8 & $-6,4$ \\
\hline Chocó & 34,4 & 37,5 & 1,5 \\
\hline Huila & 16,6 & 12,2 & $-5,1$ \\
\hline La Guajira & 23,0 & 20,9 & $-1,6$ \\
\hline Magdalena & 24,5 & 17,6 & $-5,4$ \\
\hline Meta & 15,7 & 15,8 & 0,1 \\
\hline Nariño & 17,3 & 15,0 & $-2,3$ \\
\hline Norte de Santander & 22,8 & 13,3 & $-8,6$ \\
\hline Quindío & 13,7 & 15,8 & 2,4 \\
\hline Risaralda & 14,5 & 12,5 & $-2,5$ \\
\hline Santander & 12,0 & 10,4 & $-2,4$ \\
\hline Sucre & 12,6 & 10,9 & $-2,3$ \\
\hline Tolima & 15,6 & 11,1 & $-5,6$ \\
\hline Valle del Cauca & 13,4 & 11,4 & $-2,6$ \\
\hline Arauca & 12,6 & 10,0 & $-3,8$ \\
\hline Casanare & 14,4 & 12,2 & $-2,7$ \\
\hline Putumayo & 19,6 & 13,0 & $-6,6$ \\
\hline San Andrés y Providencia & 12,1 & 13,8 & 2,1 \\
\hline Amazonas & 20,4 & 32,9 & 8,4 \\
\hline Guainía & 40,6 & 47,2 & 2,6 \\
\hline Guaviare & 16,3 & 13,9 & $-2,6$ \\
\hline Vaupés & 44,4 & 35,6 & $-3,6$ \\
\hline Vichada & 12,3 & 43,6 & 23,5 \\
\hline Media & 17,0 & 13,6 & $-3,7$ \\
\hline Desviación estándar & 4,0 & 3,9 & $-0,2$ \\
\hline Coeficiente de variación & 0,2 & 0,3 & 3,6 \\
\hline
\end{tabular}

Nota:los estadísticos estimados corresponden a media y desviación estándar ponderadas. La base de ponderación es el número de nacidos vivos.

Fuente: cálculos propios con base en datos del Departamento Administrativo Nacional de Estadística.

tasa de descenso anual de $8,6 \%$, en el departamento de Norte de Santander, a tasas anuales de incremento, como se observa para el departamento de Vichada. Estas variaciones regionales parecen persistir como sugiere el incremento de la desviación estándar y del coeficiente de variación estadística (estos resultados pueden ser mayores debido a la mejora en el registro en las regiones de bajos ingresos).

Respecto a condiciones socioeconómicas y de cobertura en servicios de salud (Tabla 2) es importante resaltar que las disparidades socioeconómicas se estrecharon un poco, mientras que el acceso a una mejor atención en salud se amplió.

La TMI departamental en Colombia se relaciona negativamente con el porcentaje de madres con educación secundaria y superior, así como con el ingreso (Figura 1.A y 1.B) -válido para el ingreso per cápita, los resultados pueden ser obtenidos de los autores-. Mientras que la estimación no significativa en 2003 puede sugerir que hay problemas en los datos, el impacto de los coeficientes de educación para los años 2006 y 2009 no son estadísticamente diferentes entre sí, sugiriendo que un aumento del $10 \%$ en la proporción de madres con educación secundaria reduce la TMI promedio para Colombia en cerca de $6 \%$ (Tabla 3, ecuación 1). Los ingresos tienen un efecto similar (ecuación 2), un aumento del $10 \%$ del promedio de ingresos y se asocia con una disminución del $5 \%$ en la TMI promedio.

La TMI departamental está negativamente relacionada con el porcentaje de madres afiliadas al RC (Figura 1.C), así como con el porcentaje de madres atendidas por médico durante el parto o en el episodio que lleva a la muerte del niño y el porcentaje de cesáreas. El impacto relativo del aseguramiento se incrementa en magnitud y significancia estadística en el tiempo; este se duplica en los 6 años del estudio (Tabla 3, ecuación 3). Mientras que en 2003, un aumento del $10 \%$ en la proporción de afiliaciones en el RC produce una caída del $2,6 \%$ de la TMI promedio, en 2009 el descenso es de 5,5\%. El efecto del uso de los servicios, representados por el porcentaje de madres que fueron atendidas por un médico y quienes tuvieron un parto por cesárea, un indicativo de accesibilidad a calidad de servicios, también contribuyen a la reducción de la TMI. Aquí los resultados son robustos en cuanto se aumenta en el tiempo.

Tabla 2. Nivel y variación en nivel de educación, ingreso per cápita y cobertura de seguridad en salud, 2003-2009

\begin{tabular}{|c|c|c|c|c|c|c|c|c|c|}
\hline \multirow[t]{2}{*}{ Variable } & \multicolumn{2}{|c|}{$\begin{array}{c}\text { Madres con educación } \\
\text { secundaria }(\%)\end{array}$} & \multirow{2}{*}{$\begin{array}{l}\text { TVA } \\
(\%)\end{array}$} & \multicolumn{2}{|c|}{$\begin{array}{c}\text { PIB per cápita } \\
\text { (millones de COP) }\end{array}$} & \multirow{2}{*}{$\begin{array}{l}\text { TVA } \\
(\%)\end{array}$} & \multicolumn{2}{|c|}{$\begin{array}{c}\text { Madres en } \\
\text { contributivo (\%) }\end{array}$} & \multirow{2}{*}{$\begin{array}{l}\text { TVA } \\
(\%)\end{array}$} \\
\hline & 2003 & 2009 & & 2003 & 2009 & & 2003 & 2009 & \\
\hline Media & 37,7 & 43,7 & 2,5 & 7,4 & 9,1 & 3,5 & 35,3 & 40,2 & 2,2 \\
\hline Coeficiente de variación & 0,2 & 0,2 & & 0,5 & 0,4 & & 0,4 & 0,4 & \\
\hline
\end{tabular}

TVA: tasa de variación anual, COP: pesos colombianos

Nota: los estadísticos estimados corresponden a media y desviación estándar ponderadas. La base de ponderación es el número de nacidos vivos, excepto para el PIB per cápita que usa la población total del departamento (Censo 2005).

Fuente: cálculos propios con base en datos del Departamento Administrativo Nacional de Estadística. 

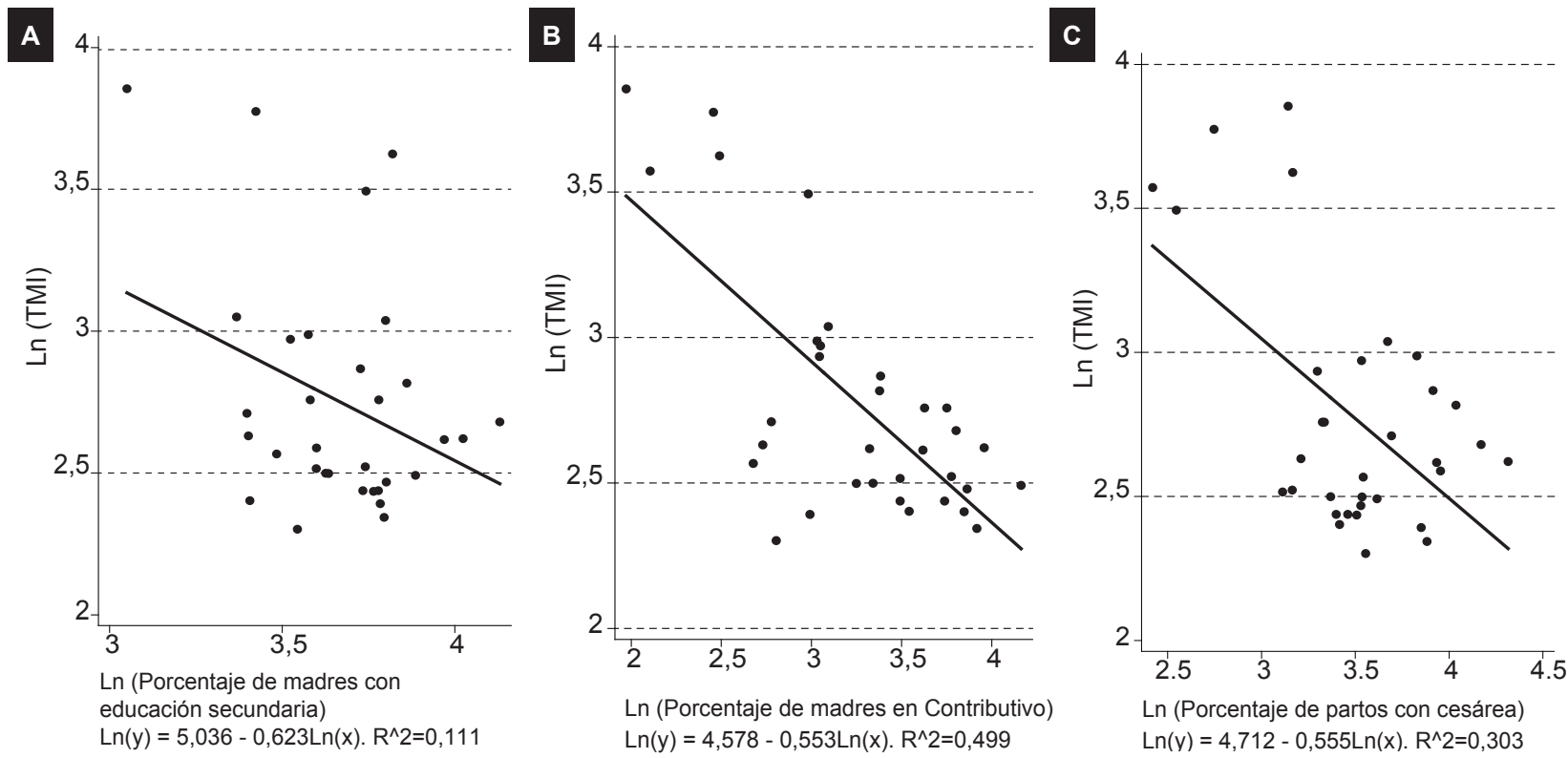

Figura 1. Tasa de mortalidad infantil departamental frente a condiciones socioeconómicas, acceso a aseguramiento y uso de intervenciones médicas (2009)

TMI: tasa de mortalidad infantil.

A: Ln (TMI) frente a Ln (porcentaje de madres con educación secundaria); B: Ln (TMI) frente a Ln (porcentaje de madres en contributivo); C: Ln (TMI) frente a Ln (porcentaje de partos por cesárea).

Fuente: cálculos propios con base en datos del Departamento Administrativo Nacional de Estadística.

El nivel de ajuste de las regresiones presentadas en la Figura 1, indica que la relación (negativa) más fuerte es entre la TMI y la vinculación al RC $(0,49)$; le siguen la relación con el uso de cesáreas $(0,30)$ y nivel de educación $(0,11)$.
El número total $\mathrm{CMI}$ por cada mil NV no tiene una asociación con la TMI (Tabla 4, ecuación 1). La disminución de la TMI se asocia, sin embargo, con la disponibilidad de CMI privadas por cada mil NV y el porcentaje de camas de cuidado intensivo infantil (Tabla 4 , ecuaciones 2 y 3 ).

Tabla 3. Efecto de las condiciones demográficas y socioeconómicas y de acceso a la seguridad social sobre la tasa de mortalidad infantil departamental (estimados separados por año)

\begin{tabular}{|c|c|c|c|c|c|c|}
\hline \multirow{4}{*}{ Año } & \multirow{4}{*}{$\begin{array}{l}\text { Variables } \\
\text { independientes }\end{array}$} & \multicolumn{5}{|c|}{$\begin{array}{l}\text { Variable dependiente: Ln TMI departamental } \\
\text { (|t-stat| en paréntesis) }\end{array}$} \\
\hline & & \multicolumn{2}{|c|}{$\begin{array}{l}\text { Condiciones demográficas y } \\
\text { socioeconómicas }\end{array}$} & \multicolumn{3}{|c|}{$\begin{array}{c}\text { Acceso a seguridad social y uso de intervenciones } \\
\text { médicas }\end{array}$} \\
\hline & & $\begin{array}{l}\text { Ln (Porcentaje de } \\
\text { madres con educación } \\
\text { secundaria) }\end{array}$ & $\begin{array}{l}\text { Ln (PIB per } \\
\text { cápita) }\end{array}$ & $\begin{array}{l}\text { Ln (Porcentaje } \\
\text { de madres en } \\
\text { Contributivo) }\end{array}$ & $\begin{array}{l}\text { Ln (Porcentaje de } \\
\text { madres atendidas } \\
\text { por médico) }\end{array}$ & $\begin{array}{l}\text { Ln (Porcentaje } \\
\text { de partos por } \\
\text { cesárea) }\end{array}$ \\
\hline & & $\mathrm{N}=32^{\mathrm{a}}$ & $\mathrm{N}=32^{\mathrm{b}}$ & $\mathrm{N}=33$ & $\mathrm{~N}=32^{\mathrm{c}}$ & $\mathrm{N}=33$ \\
\hline \multirow{3}{*}{ ¿̊ } & Coeficiente & $\begin{array}{l}-0,19 \\
(1,12)\end{array}$ & $\begin{array}{l}-0,44^{*} \\
(4,04)\end{array}$ & $\begin{array}{c}-0,26^{* *} \\
(2,49)\end{array}$ & $\begin{array}{c}-0,99^{* *} \\
(1,99)\end{array}$ & $\begin{array}{c}-0,32^{* *} \\
(2,44)\end{array}$ \\
\hline & Constante & $\begin{array}{l}3,53^{*} \\
(6,11)\end{array}$ & $\begin{array}{l}9,70^{*} \\
(5,78)\end{array}$ & $\begin{array}{c}3,74^{*} \\
(11,14)\end{array}$ & $\begin{array}{l}7,36^{*} \\
(3,26)\end{array}$ & $\begin{array}{l}3,93^{*} \\
(9,34)\end{array}$ \\
\hline & $\mathbf{R}^{2}$ & 0,04 & 0,35 & 0,17 & 0,12 & 0,16 \\
\hline \multirow{4}{*}{ ஜ } & Coeficiente & $-0,59^{*}$ & $-0,54^{*}$ & $-0,46^{*}$ & $-2,72^{*}$ & $-0,49^{*}$ \\
\hline & & $(4,07)$ & $(5,83)$ & $(4,91)$ & $(2,85)$ & $(3,93)$ \\
\hline & Constante & $4,97^{\star}$ & $11,30^{*}$ & $4,36^{*}$ & $15,23^{*}$ & $4,53^{*}$ \\
\hline & $\mathbf{R}^{2}$ & $\begin{array}{l}(9,60) \\
036\end{array}$ & $(7,84)$ & $(14,46)$ & $(3,52)$ & $(10,84)$ \\
\hline \multirow{5}{*}{ ஓ् } & Coeficiente & $\begin{array}{l}0,36 \\
-0,62+\end{array}$ & 0,53 & 0,44 & 0,21 & 0,33 \\
\hline & & $\begin{array}{l}-0,02 \\
(1,94)\end{array}$ & $(4,53)$ & $\begin{array}{l}-0,50 \\
(5,56)\end{array}$ & $\begin{array}{l}-5,4 y \\
(582)\end{array}$ & $\begin{array}{l}-0,50 \\
(367)\end{array}$ \\
\hline & Constante & $5,04^{*}$ & $12,13^{*}$ & $4,58^{*}$ & $27,81^{*}$ & $4,71^{*}$ \\
\hline & & $(4,26)$ & $(5,88)$ & $(13,93)$ & $(6,46)$ & $(8,86)$ \\
\hline & $\mathbf{R}^{2}$ & 0,11 & 0,41 & 0,50 & 0,53 & 0,30 \\
\hline
\end{tabular}

TMI: tasa de mortalidad infantil; Ln: logarítmo natural; * ${ }^{* *}$ y ${ }^{\dagger}$ : significativo al 1,5 y $10 \%$, respectivamente

${ }^{a}$ No incluye departamento de Vaupés; ${ }^{b}$ Pesos colombianos a precios corrientes. No incluye departamento de Casanare; ${ }^{c}$ No incluye departamento de Vaupés

Fuente: cálculos propios con base en datos del Departamento Administrativo Nacional de Estadística. 
Tabla 4. Efecto de la disponibilidad de servicios de salud sobre la tasa de mortalidad infantil departamental (estimados separados por año)

\begin{tabular}{|c|c|c|c|c|}
\hline \multirow{3}{*}{ Año } & \multirow{3}{*}{$\begin{array}{c}\text { Variables } \\
\text { independientes }\end{array}$} & \multicolumn{3}{|c|}{ Variable dependiente: Ln TMI departamental ( |t-stat| en paréntesis) } \\
\hline & & Ln (Total CMI por mil NV) & Ln (CMI privadas por mil NV) & Ln (Porcentaje de CPN de cuidado intensivo) \\
\hline & & $\mathrm{N}=33^{\mathrm{a}}$ & $\mathrm{N}=33$ & $\mathrm{~N}=33$ \\
\hline \multirow{4}{*}{ ஜ્స } & Coeficiente & $-0,06$ & $-0,03$ & $-0,06^{\dagger}$ \\
\hline & & $(1,20)$ & $(0,58)$ & $(1,66)$ \\
\hline & Constante & $\begin{array}{c}3,00^{*} \\
(31,22)\end{array}$ & $\begin{array}{c}2,94^{*} \\
(37,57)\end{array}$ & $\begin{array}{c}3,02^{*} \\
(34,65)\end{array}$ \\
\hline & $\mathbf{R}^{2}$ & 0,01 & 0,01 & $\begin{array}{c}0,08 \\
0,03)\end{array}$ \\
\hline \multirow{5}{*}{ ঃ } & Coeficiente & 0,01 & $-0,15^{*}$ & $-0,15^{\star}$ \\
\hline & & $(0,89)$ & $(2,80)$ & $(3,39)$ \\
\hline & Constante & $2,87^{*}$ & $3,09^{*}$ & $3,22^{*}$ \\
\hline & & $(13,51)$ & $(35,18)$ & $(29,48)$ \\
\hline & $\mathbf{R}^{2}$ & 0,01 & 0,20 & 0,27 \\
\hline \multirow{5}{*}{ : } & Coeficiente & 0,55 & $-0,14^{* *}$ & $-0,16^{*}$ \\
\hline & & $(1,55)$ & $(2,01)$ & $(2,82)$ \\
\hline & Constante & 0,93 & $3,03^{*}$ & $3,14^{*}$ \\
\hline & & $(0,78)$ & $(20,79)$ & $(21,51)$ \\
\hline & $\mathbf{R}^{2}$ & 0,08 & 0,12 & 0,20 \\
\hline
\end{tabular}

TMI: tasa de mortalidad infantil; CMI: camas materno infantil; CPN: camas pediátricas y neonatales; NV: nacidos vivos; Ln: logaritmo natural; *, ** ${ }^{+}$: significativo al 1,5 y $10 \%$, respectivamente

a No incluye departamentos de Arauca ni San Andrés y Providencia para 2009

Fuente: cálculos propios con base en datos del Departamento Administrativo Nacional de Estadística.

Los efectos negativos de la oferta de CMI privadas sobre la TMI parecen permanecer constantes entre los años 2006 y 2009, mientras que el impacto asociado al porcentaje de camas de cuidado intensivo infantil parece crecer durante el período.
Las estimaciones del sistema de ecuaciones estructurales están resumidas en la Tabla 5. Más atenciones por médico (ecuación 1), el indicador de atención médica y uso, tiene un efecto significativo sobre la reducción de la TMI. El nivel de educación

Tabla 5. Sistemas de ecuaciones estructurales (efectos estructurales) para mortalidad infantil en Colombia

\begin{tabular}{|c|c|c|c|c|c|c|}
\hline \multirow{3}{*}{ Variables independientes } & \multicolumn{6}{|c|}{ Variables dependientes ( |t-stat| en paréntesis) } \\
\hline & TMI & $\begin{array}{c}\text { Porcentaje } \\
\text { de partos por } \\
\text { cesárea }\end{array}$ & $\begin{array}{l}\text { Porcentaje de } \\
\text { madres atendidas } \\
\text { por médico }\end{array}$ & $\begin{array}{l}\text { Porcentaje } \\
\text { de madres en } \\
\text { contributivo }\end{array}$ & $\begin{array}{l}\text { Total CMI } \\
\text { por mil NV }\end{array}$ & $\begin{array}{c}\text { Porcentaje de } \\
\text { CMI privadas } \\
\text { por mil NV }\end{array}$ \\
\hline & (eq.1) & (eq.2) & (eq.3) & (eq.4) & (eq.5) & (eq.6) \\
\hline \multicolumn{7}{|l|}{ Variables endógenas } \\
\hline TMI & & & & & $\begin{array}{l}-0,56^{*} \\
(2,95)\end{array}$ & \\
\hline Porcentaje de partos por cesárea & $\begin{array}{l}0,95^{\star *} \\
(2,19)\end{array}$ & & & & & \\
\hline $\begin{array}{l}\text { Porcentaje de madres en contri- } \\
\text { butivo }\end{array}$ & & & & & $\begin{array}{l}-0,26^{*} \\
(3,05)\end{array}$ & $\begin{array}{l}1,16^{*} \\
(2,67)\end{array}$ \\
\hline Total CMI por mil NV & & $\begin{array}{l}0,94^{*} \\
(3,61)\end{array}$ & $\begin{array}{l}0,73^{*} \\
(3,22)\end{array}$ & & & \\
\hline $\begin{array}{l}\text { Porcentaje de madres atendidas } \\
\text { por médico }\end{array}$ & $\begin{array}{l}-1,77^{*} \\
(4,27)\end{array}$ & & & & & \\
\hline $\begin{array}{l}\text { Porcentaje de CMI privadas por } \\
\text { mil NV }\end{array}$ & & $\begin{array}{l}0,62^{*} \\
(4,23)\end{array}$ & $\begin{array}{l}0,73^{*} \\
(6,11)\end{array}$ & & & \\
\hline \multicolumn{7}{|l|}{ Variables exógenas } \\
\hline Edad media de la madre & & $\begin{array}{c}0,85 \\
(0,70)\end{array}$ & & & & \\
\hline $\begin{array}{l}\text { Porcentaje de madres con educa- } \\
\text { ción secundaria }\end{array}$ & $\begin{array}{l}-0,06 \\
(0,16)\end{array}$ & $\begin{array}{l}0,36^{*} \\
(3,01)\end{array}$ & & & & $\begin{array}{l}-0,46 \\
(1,02)\end{array}$ \\
\hline PIB per cápita (millones) & & & & $\begin{array}{l}1,32^{*} \\
(7,42)\end{array}$ & & \\
\hline Tiempo & & & & $\begin{array}{c}0,47 \\
(1,23)\end{array}$ & $\begin{array}{c}3,43^{*} \\
(11,63)\end{array}$ & \\
\hline Constante & $\begin{array}{c}155,94^{*} \\
(5,21)\end{array}$ & $\begin{array}{c}-48,48^{\dagger} \\
(1,65)\end{array}$ & $\begin{array}{l}46,77^{*} \\
(5,06)\end{array}$ & $\begin{array}{l}18,83^{*} \\
(13,04)\end{array}$ & $\begin{array}{l}35,57^{*} \\
(6,46)\end{array}$ & $\begin{array}{l}29,36 * \\
(3,88)\end{array}$ \\
\hline $\mathbf{N}$ & 230 & 230 & 230 & 230 & 230 & 230 \\
\hline Chi-sq & $35,00 *$ & $198,50 *$ & $60,70^{*}$ & $145,75^{*}$ & $58,96^{*}$ & $20,55^{*}$ \\
\hline$R^{2}$-Ajustado & $-3,16$ & $-1,10$ & $-4,23$ & 0,18 & 0,20 & $-0,02$ \\
\hline Harvey LM Test ${ }^{+\dagger}$ & $9,59^{*}$ & 0,41 & 1,80 & $13,18^{*}$ & $4,48^{* *}$ & 0,97 \\
\hline
\end{tabular}

TMI: tasa de mortalidad infantil; CMI: camas materno infantil; NV: nacidos vivos

eq.: ecuación; * ${ }^{* *} \mathrm{y}^{\dagger}$ : significativo al 1,5 y $10 \%$, respectivamente; ${ }^{\dagger+}$ : el estadístico del Harvey LM Test global es 30,4 , significativo al $1 \%$.

Fuente: cálculos propios con base en datos del Departamento Administrativo Nacional de Estadística. 
no tiene efecto significativo sobre M. El efecto positivo significativo de la cirugía cesárea, puede estar asociado con la necesidad de este procedimiento en madres de alto riesgo, según lo sugerido por otros autores ${ }^{(13)}$. Las cirugías cesáreas (ecuación 2) están, relativamente hablando, fuertemente afectadas por la oferta de camas, principalmente privadas. El nivel de educación también incrementa significativamente la demanda por el procedimiento. Sin embargo, estadísticamente se encuentra que, la edad de la madre no tiene efecto. Al igual que los partos por cesárea, la atención por médico (ecuación 3) se ve afectada positivamente, por la infraestructura, medida por la oferta de CMI, privada y total. El RC de aseguramiento (ecuación 4) está positivamente asociado con el ingreso entre los departamentos y en el tiempo, aunque no con el desarrollo $(T)$, puesto que el coeficiente respectivo no es significativamente diferente de cero. La oferta total de CMI (ecuación 5) está afectada positivamente por el desarrollo general $(\mathrm{T})$ y negativamente por ambos, el acceso a mejor aseguramiento y la TMI. Además, el porcentaje de CMI privadas (ecuación 6) está positivamente afectado por el acceso al aseguramiento en el RC, sugiriendo mayor accesibilidad a recursos privados, donde hay mayor población afiliada a seguros privados de salud. Mientras tanto, el nivel de educación no ha tenido efectos sobre la disponibilidad de este tipo de camas.

\section{DISCUSIÓN}

La TMI en Colombia entre 2003 y 2009 tuvo una reducción superior a la reportada por Alarcón et al. en 2006 en la década anterior ${ }^{(5)}$. El deterioro observado en la TMI en algunos departamentos, donde en realidad aumentó, se asocia a los datos de registro inconsistentes: en Vichada, Amazonas, Guainía, Meta, Chocó (1,12). Todos son departamentos pobres con una historia de subregistro relativamente alta, especialmente en el número de niños fallecidos en 2003, pero que presentan una rápida mejora en el reporte de estadísticas vitales para el año 2009 (1). Por esto, podemos suponer que en el estudio, el período anterior tuvo disparidades, en realidad más grandes que las reportadas. Las diferencias pueden ir disminuyendo pero aún persisten, a pesar de ciertas mejoras en las condiciones socioeconómicas. Esta conclusión es consistente con recientes publicaciones de Urdinola en 2011 y con hallazgos de otros países ${ }^{(12-16)}$.

La clave es que los departamentos con mejor nivel -también en términos de datos-, como Santander, Valle del Cauca, Antioquia, Cundinamarca y Bogotá (Tabla 1), tienen una TMI cercana a la de países con los niveles más bajos de Latinoamérica y El Caribe (2009-2010), como Chile $(7,9)$, Costa Rica $(9,0)$. Uruguay $(9,0)$; mientras que otros departamentos que presentan TMI más altas, como Guainía $(47,2)$, Vichada $(43,6)$, Chocó $(37,5)$, Vaupés $(35,6)$ y Amazonas $(32,9)$, están cercanos a Bolivia $(42,0)$ y podrían estar por encima de Guatemala $(25,0)$ y Nicaragua $(23,0)$. Una tercera parte de los departamentos presentan tasas superiores al promedio de América Latina y el Caribe para el año $2009(18,0)^{(17,18)}$.

Las relativamente grandes disparidades en Colombia potencian el problema acerca de la relativa importancia de las condiciones socioeconómicas frente a la atención en salud en la determinación de la TMI en todo el país; tal como se ha presentado en la literatura reciente ${ }^{(19)}$. Una hipótesis plausible es que a medida que las condiciones económicas mejoran y disminuyen las tasas de mortalidad infantil, la tecnología médica empieza a cobrar relativa importancia, puesto que los efectos de las condiciones socioeconómicas tienden a desvanecerse (desnutrición, baja adherencia al control prenatal y posnatal, etc.), reduciendo su impacto, mientras que la importancia relativa de la atención médica se eleva. Esta hipótesis se apoya también en la realidad en que cuando la TMI va disminuyendo, se requieren esfuerzos médicos cada vez mayores para salvar la vida de los bebés, principalmente relacionados con la supervivencia de niños prematuros, con anomalías congénitas o con complicaciones del embarazo y del parto, como la hemorragia en la madre y la preeclampsia-eclampsia. Medidas que pueden mejorar el pronóstico del bebé contando con oportunidad de acceso a atención médica de calidad y recursos tecnológicos de cuidado intensivo ${ }^{(20)}$. Entonces, cuando las TMI disminuyen, el acceso a los servicios se hace cada vez más importante en comparación con las condiciones socioeconómicas.

De hecho, los resultados del análisis bivariado acerca de las correlaciones de la TMI entre los departamentos, a través del tiempo, apoyan esta hipótesis (Tabla 3). Estos hallazgos sugieren que el impacto de la mejora en el aseguramiento -que también está asociado con una mejor calidad de atención (camas privadas en lugar de camas totales)- parece eclipsar, con el tiempo, el impacto de las mejores condiciones socioeconómicas. En otras palabras, el impacto del aseguramiento y el acceso potencial a los servicios de salud incrementa con el tiempo.

Aun así, el impacto único de la atención médica frente a las condiciones socioeconómicas sigue siendo un desafío, como lo sugieren las correlaciones entre las variables clave, donde se observa que la TMI está más fuertemente relacionada con la pertenencia al RC y ser atendido por un médico, que a los ingresos o la 
educación. La correlación entre la variables educación y pertenecer al RC, es de 0,76. Además, la correlación entre la proporción de partos asistidos por cesárea y el porcentaje de madres en el RC y las que fueron atendidas por un médico son 0,55 y 0,47 respectivamente.

Este desafío se corresponde -en cierta medida-con las estimaciones multivariadas, mediante las ecuaciones estructurales, que es más claro que con las estimaciones bivariadas y sustenta la conclusión acerca de la creciente importancia de la mejora del aseguramiento y la atención médica. De otro lado, las estimaciones sugieren que la atención médica, ya sea en el nacimiento o al momento de la muerte, es ampliamente superior al efecto de la educación. Más importante aun es el impacto del nivel de ingresos promedio (asociado con el nivel educativo), a través del aseguramiento y de la prestación de servicios y no a través de un efecto directo (de la educación). La infraestructura -en función del desarrollo económico y no en función de las necesidades- es importante también, por la disponibilidad de médicos y una mejor atención de calidad, medida por CMI privadas.

A pesar del desafío estadístico, basado en el análisis de las variaciones departamentales de la mortalidad infantil en Colombia entre 2003 y 2009, se concluye que el impacto del acceso a una atención de calidad, es superior al impacto de las condiciones socioeconómicas. En este estudio se aborda una cuestión política clave para Colombia y otros países: el impacto relativo y cambiante de la atención médica, en comparación con las condiciones socioeconómicas sobre la mortalidad infantil. En este sentido, como estas condiciones mejoran con el desarrollo general, el acceso a la atención se vuelve aun más importante. Adicionalmente, parece indicar que la oferta de CMI y recursos relacionados con la medicina en Colombia no responde a las tasas de mortalidad infantil, sino más bien a los ingresos y al desarrollo económico. De acuerdo con esto, las medidas de política pública en Colombia deben orientarse a intervenir más agresivamente la TMI, aumentando los recursos donde la mortalidad infantil es más alta.

Agradecimientos: a Juan David Martin, asistente de investigación, por sus aportes y procesamiento de la información estadística.

Contribuciones de autoría: MJM participó de la concepción y diseño del estudio, organizó las bases de datos y redactó el artículo. DC participó del diseño de la metodología y del análisis estadístico de los datos, así como de la revisión en todo momento de resultados, discusión y conclusiones. JJM participó de la revisión crítica y corrección del artículo. Todos los autores aprobaron la versión final.

Fuentes de financiamiento: recursos de investigaciones de la Universidad Icesi. Las bases de datos provienen del Sistema de Estadísticas vitales del Departamento Administrativo Nacional de Estadística (DANE) en Colombia, suministradas a la Universidad Icesi, mediante Convenio N. ${ }^{\circ}$ 014-2008 (Licencia de uso de información de muertes y nacimientos en los períodos 1979-2009 y 1998-2009). Este artículo ha sido beneficiado del soporte general del Banco Mundial en el Estudio de descentralización de sistema de salud en Colombia.

Conflictos de interés: los autores declaran no tener conflictos de interés.

\section{REFERENCIAS BIBLIOGRÁFICAS}

1. Jaramillo-Mejía M, Chernichovsky D, Jiménez-Moleón JJ. Resolving the enigma of infant mortality rate in Colombia [Internet]. Rochester, NY: Social Science Research Network; 2012 [citado el 21 de abril de 2013]. Report No.: ID 2096901. Available from: http://papers.ssrn.com/ abstract $=2096901$

2. Colombia, Ministerio de Salud y Protección Social. Indicadores Básicos 2010: Situación de Salud de Colombia [Internet]. Bogotá: Ministerio de Salud y Protección Social; 2011 [citado el 21 de abril de 2013]. Disponible en: http:// www.minsalud.gov.co/salud/Paginas/ INDICADORESBASICOSSP.aspx

3. Colombia, Departamento Administrativo Nacional de Estadística (DANE). Estimaciones Tasa de Mortalidad infantil nacional, departamen- tal y municipal, período 2005-2009 [Internet]. Bogotá: DANE; 2011 [citado el 21 de abril del 2013]. Disponible en: http://www.dane.gov.co

4. Organización Panamericana de la Salud. Situación de salud en las Américas: Indicadores Básicos 2012 [Internet]. Geneva: OPS; 2012 [citado el 21 de abril de 2013]. Disponible en: http://new.paho.org/hq/index. php?option $=$ com_content\&view $=$ arti cle\&id=7170\&Itemid=2395\&lang=es

5. Alarcón González DA, Robles M. Los retos para medir la mortalidad infantil con registros civiles incompletos. Washington, DC: Inter-American Development Bank; 2007.

6. Colombia, Departamento Administrativo Nacional de Estadística (DANE). Proyecciones nacionales, departamentales y de población 2005 -
2020 [Internet]. Bogotá: DANE; 2007. Report No. 7.

7. Congreso de la República de Colombia. Ley 100 de 1993. Por medio de la cual se crea el Sistema General de Seguridad Social Integral. Libro II. Sistema General de Seguridad Social en Salud. Ley 100 Bogotá, diciembre de, 1993.

8. Montenegro F, Bernal O. Colombia Case Study: The Subsidized Regime of Colombia's National Health Insurance System [Internet]. Washington DC: The World Bank; 2013 [citado el 28 de abril de 2013]. Disponible en: http:// www-wds.worldbank.org/external/ default/WDSContentServer/WDSP/ IB/2013/02/01/000425962_201302 01114946/Rendered/PDF/749610N WP0COLO00Box374316B00PUBL IC0.pdf 
9. Chernichovsky D, Guerrero R, Martínez G. La sinfonía incompleta: La reforma del Sistema de Salud de Colombia. [Internet]. Working Paper. Centro de Estudios en Protección Social y Economía de la Salud; 2012. Disponible en: http://www. proesa.org.co/proesa/images/docs/ The\%20Incomplete\%20Symphony_ Span_27Sep12.pdf

10. Colombia, Departamento Administrativo Nacional de Estadística (DANE). PIB cuentas departamentales [Internet]. Bogotá: DANE; c2013 [citado el 21 de abril del 2013]. Disponible en: http://www.dane.gov. co/index.php/pib-cuentas-nacionales/ cuentas-departamentales

11. Colombia, Ministerio de Salud y Protección Social. Registro Especial de prestadores de Servicios de Salud (REPS) [Internet]. Bogotá: Ministerio de Salud y Protección Social; c2013. Disponible en: http://201.234.78.38/ habilitacion/

12. Urdinola BP. Determinantes socioeconómicos de la mortalidad infantil en Colombia, 1993. Rev Colomb Estad. 2011;34(1):39-72.
13. Lauer JA, Betrán AP, Merialdi M, Wojdyla D. Determinants of caesarean section rates in developed countries: supply, demand and opportunities for control. World Health Rep 2010 Backgr Pap [Internet]. Geneva: WHO; 2010.

14. Arroyave I, Cardona D, Burdorf A, Avendano M. The impact of increasing health insurance coverage on disparities in mortality: health care reform in Colombia, 1998-2007. Am J Public Health. 2013;103(3):e100-6. doi: 10.2105/AJPH.2012.301143.

15. Dallolio L, Gregori VD, Lenzi J, Franchino G, Calugi S, Domenighetti $\mathrm{G}$, et al. Socio-economic factors associated with infant mortality in Italy: an ecological study. Int J Equity Health. 2012;11(1):45. doi: 10.1186/1475-9276-11-45.

16. Laskar MS, Harada N. Trends and regional variations in infant mortality rates in Japan, 1973-1998. Public Health. 2005;119(7):659-63.

17. UNICEF. El Estado Mundial de la Infancia de 2012: Niñas y niños en un mundo urbano [Internet]. UNICEF; 2012 [citado el 8 de setiembre de 2013].
Disponible en: http://www.unicef.org/ spanish/sowc2012/

18. Kaempffer RA, Medina LE. Mortalidad infantil reciente en Chile: Éxitos y desafíos. Rev Chil Pediatr. 2006;77(5):492-500.

19. Schell CO, Reilly M, Rosling $\mathrm{H}$, Peterson S, Ekström AM. Socioeconomic determinants of infant mortality: a worldwide study of 152 low-, middle-, and high-income countries. Scand J Public Health. 2007;35(3):288-97.

20. Campos-Miño S, Sasbón JS, von Dessauer B. Los cuidados intensivos pediátricos en Latinoamérica. Med Intensiva. 2012;36(1):3-10.

Correspondencia: Marta Cecilia Jaramillo-Mejia Dirección: Facultad de Ciencias Administrativas y Económicas, Universidad Icesi, Calle 18 \# 122-135 Pance, oficina \# 1523. Cali, Colombia

Teléfono: +57-2-5552334 Ext. 8433, +57 3154898845 .

Correoelectrónico:mcjara@icesi.edu.co

\section{Consulte las ediciones anteriores de la Revista Peruana de Medicina Experimental y Salud Pública en} wWw.scielosp.org

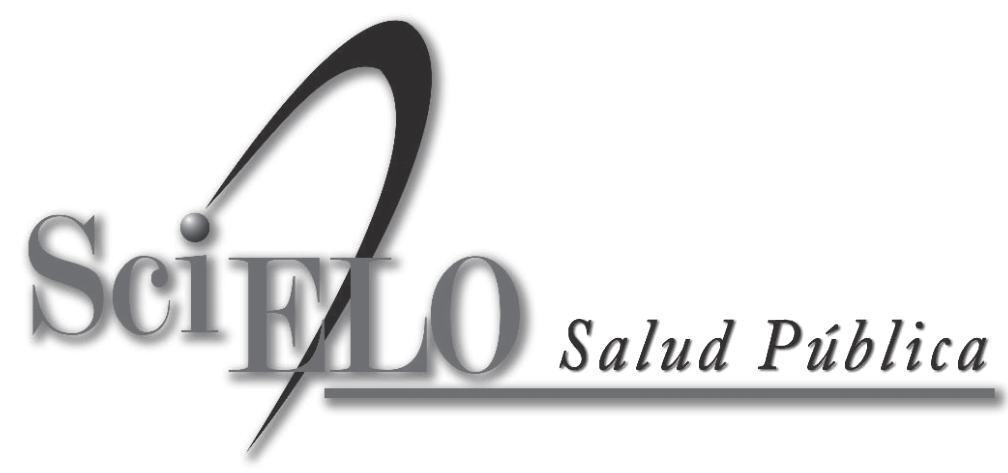

